\title{
Jean-Jacques Déthier
}

Militärputsch in der Türkei

Interview mit einem türkischen Genossen

Am 12. September 1980 um 4 Uhr morgens verkündete ein über den Rundfunk verlesenes Communiqué, daß in der Türkei die Armee unter Führung von Generalstabschef General $\mathrm{K}$.Evren die Macht ergriffen und zur Ausübung ihrer Herrschaft einen 'Nationalen Sicherheitsrat' gebildet hat, daß Regierung und Parlament aufgelöst sind und daß im ganzen Lande das Kriegstecht ausgerufen ist. Dieser Staatsstreich wurde binnen kurzem von allen westlichen Ländern begrüßt, insbesondere von den USA und Westdeutschland, auch in Moskau hatte er ein recht positives Echo.

Nach den Worten der Militärs sollte der Putsch den Zustand eines 'faktischen Bürgerkriegs' beenden, die nationale Einheit retten, die Sicherheit der Bürger schützen und die Autorität des Staates wiederherstellen, der durch politische Unruhen und eine tiefgehende Wirtschaftskrise bedroht war. Ausländische Zeitungen sahen in dem Putsch übereinstimmend den mutigen Versuch, Recht und Ordnung in einem Lande wiederherzustellen, das seit dem Ende des Zweiten Weltkriegs ein sehr stürmisches politisches Leben hinter sich hat. Alle Jornalisten wiesen darauf hin, daß die Türken wenigstens »das erste ruhige Wochenende seit vielen Jahren« verbracht hätten (Le Monde vom 16.9.80). Und nur sehr wenige sahen in dem Putsch die Fortsetzung und Zuspitzung der Repression, der die Linke seit der Ausrufung des Kriegsrechts im Dezember 1979 ausgesetzt gewesen war.

Dieses Interview mit einem türkischen Genossen aus Istanbul wurde eine Woche nach dem Putsch in einem Nahost-Land gemacht. Darin wird versucht, die Gründe für die Machtübernahme der Militärs zu erklären und Elemente für eine Analyse der gegenwärtigen Situation in einem Lande herauszuarbeiten, das für die Strategie des westlichen Imperialismus eine Schlüsselrolle spielt.

\section{War die Linke auf den Putsch vorbereitet?}

Ich glaube nicht. Niemand in der Türkei erwartete eigentlich einen Putsch zu dieser Zeit. Das Militär hatte die Demirel-Regierung und das Parlament mehrfach in den vergangenen Monaten gewarnt, aber irgendwie war - so ironisch das klingen mag - die Drohung mit einer militärischen Machtübernahme so alltäglich, daß alle überrascht waren, als es schließlich so weit war.

Zu den Gründen, die den 'Nationalen Sicherheitsrat' zur Machtergreifung veranlaßt haben, kann man den Beschluß des Parlaments zählen, dạs kurz zuvor den Rücktritt des Außenministers erzwungen hatte, und die Haltung der kleinen, aber einflußreichen Nationalen Heilspartei (MSP) - für das traditionell auf den kemalistischen Konstitutionalismus orientierte Militär wahrlich ein rotes Tuch - oder die Tatsache, daß das Parlament seit mehr als 6 Monaten unfähig war, einen neuen Präsidenten der Republik zu wählen. Aber all dies waren nur Vorwände, welche die Armee jetzt und nicht später zum Einschreiten veranlaßt haben. Denn eigentlich hatte sich die Situation schon seit langem ständig verschlechtert.

Die Linke - und natürlich auch die offiziellen Parteien - wurden überrascht. Zumindest 
waren sie überrascht vom Ausmaß der Repression und dem radikalen Programm, das sich die Militärs vorgenommen haben. Auf der Linken nahm man allgemein an, daß das Kriegsrecht - das dem Militär bereits innerhalb des verfassungsmäßigen Rahmens extensive Macht übertrug - als Repressionsinstrument ausreichen und der herrschenden Klasse die Durchsetzung ihres ökonomischen Programms ermöglichen würde. Aber das hat sich als eine Fehleinschätzung erwiesen.

\section{Welche Unterdrückungsmaßnahmen gab es?}

Die Linke war einem Angtiff auf zwei Ebenen ausgesetzt. Zum ersten macht ihr das Kriegsrecht das Leben sehr schwer. Es wurde im Dezember 1979 ausgerufen, nach dem Massaker von Karamanmarash, als bei einem Überfall auf diese ostanatolische Stadt mehr als 400 Menschen von rechten Kommandos getötet wurden, Das Kriegsrecht wurde später auf weitere Provinzen der Türkei ausgedehnt, schließlich auf die kurdischen Landesteile und die wichtigsten Städte. Heute erstreckt es sich über das ganze Land. Kriegsrecht heißt, daß die verfassungsmäßigen Rechte aufgehoben und daß Verhaftungen und Hausdurchsuchungen leichter möglich sind. Es heißt auch, daß in den letzten Monaten 90 Prozent aller Zeitschriften der Linken und viele Bücher verboten wurden. Aber es gab immer noch eine relative Handlungsfreiheit, die jetzt völlig abgeschafft ist.

Zum zweiten wurde die Linke von rechten Gruppierungen angegriffen, die von der Regierung offiziell als 'Hilfskräfte der Sicherheitsorgane' akzeptiert waren.

\section{Offiziell?}

Ja, das war die Haltung von Demirel. Seiner Meinung nach gab es keinerlei rechten Terrorismus: es gab nur linke Terroristen auf der einen und Nationalisten auf der anderen Seite. Vor acht Monaten, kurz nachdem er seine Minderheitsregierung gebildet hatte. äußerte er ganz offen im Fernsehen: »Niemand wird mich zu der Auffassung bringen, daß es in der Türkei rechte Terroristen gibt.« Die Charakterisierung des Terrorismus - mithin die Haltung, die der Staat dazu einnehmen sollte - war auch ein Streitthema zwischen Demirel und seinem Hauptgegner Ecevit, dem Führer der Republikanischen Volkspartei (CHP). Für Ecevit gab es sowohl linken als auch rechten Terrorismus. Als er noch Ministerpräsident gewesen war, hatte er deshalb auch bei Verhaftungen ungefähr die Balance zwischen beiden Richtungen gehalten. Das heißt, so, wie es einmal ein Karrikaturist gezeichnet hat: zwei Schläge nach links und einen nach rechts. Jetzt hingegen braucht man nicht einmal mehr auf das äußere Erscheinungsbild und auf diese Art von Balance zu achten.

Demirel wußte doch aber, daß Turkesh, der Führer der Nationalen Aktionspartei (MHP), die an seiner Koalitionsregierung beteiligt war, mit den bewaffneten faschistischen Organisationen in Verbindung stand?

Natürlich wußte er das. Die Jugendorganisation der MHP (bei uns bekannt als 'Graue Wölfe', d.Ü.) gehörte doch zu diesen Organisationen. Das war überhaupt nicht zu übersehen. Nicht nur die linke Presse, auch liberale Zeitungen wie Milliyet haben diese Verbindungen Tag für Tag dokumentiert. Demirel wußte, daß das völlig illegal war. Aber da er an der Spitze einer Minderheitsregierung stand, verschloß er davor die Augen. Man kann sich sogar fragen, ob er nicht im Grunde dafür dankbar war, daß ihm die faschistischen Kommandos die Arbeit abgenommen haben, obwohl diese im Grunde so sehr außer Kontrolle gerieten, daß es vielen Leuten in Demirels Gerechtigkeitspartei (AP) - die im übrigen nic eine starke Jugendorganisation hatte - unbehaglich zu werden begann.

Was wird denn nun geschehen? Inwiefern wird sich diese neue Repressionsperiode von der des Kriegsrechts unterscheiden? 
Das ist schwer vorauszusagen. Wie schon erwähnt, gibt es unter dem Kriegsrecht eine minimale Handlungs- und Redefreiheit, die man ausnutzen kann. Damals konnten wenigstens noch zwei linke Tageszeitungen erscheinen, Demokrat und Aydinlik. Es war schwer, etwas zu veröffentlichen, aber unmöglich war es nicht. Man konnte noch seine Freunde treffen und Versammlungen abhalten, auch wenn man dabei Vorsichtsmaßnahmen treffen mußte. Die Regierung hatte Streiks für 80 Tage unrersagt, aber das galt nicht auf Dauer. Streik war noch möglich, und die Gewerkschaften waren nicht verboten.

Jetzt nach dem Pursch ist der Kampf natürlich extrem ungleich geworden. Der linke Gewerkschaftsverband DISK ist verboten, wie alle politischen Parteien und Vereinigungen. Verhaftungen, Hausdurchsuchungen und Folter nehmen enorme Ausmaße an. Die Gefängnisse waren bereits vor dem 12. September gefüllt, jetzt benutzen sie Kinos und öffentliche Gebäude, um die Leute einzusperren, zumindest vorübergehend. Jetzt werden nicht nur Aktivisten verhaftet, sondern jeder, der im Verdachr steht, eine linke Ideologie zu haben: Lehrer, Schriftsteller, Journalisten, in deren Haus man z.B. ein 'linkes' Buch findet. Es ist noch zu früh, um zu sagen, wie das neue Regime sein wird. Aber die knappen Informationen, die wir haben, lassen wenig Zweifel am Ausmaß der bevorstehenden Repression. So hat etwa der neue Staatschef General Evren in seiner ersten Pressekonferenz die »vom Staat bezahlten Lehrer und Professoren« offen beschuldigt, der Jugend staatsfeindliche Prinzipien beizubringen. Im Klartext heißt das, daß dieser Drohung praktische Schritte folgen werden und daß die neue Verfassung, an der die Junta arbeitet, die Redefreiheit abzuschaffen oder wenigstens einzuschränken versuchen wird. Ein wciteres Vorzeichen ist der Hinweis auf neue 'Staatssicherheitsgerichte', welche bei politischen Prozessen die Militärgerichte ersetzen sollen. Sie sollen in der künftigen Verfassung festgeschrieben werden und so ihren Ausnahmecharakter verlieren.

Um dic Haltung der Militärs zu verstehen, muß man auf jeden Fall im Kopf behalten, was seit 1971 geschehen ist. Denn das war für die Armee ganz sicher eine entscheidende Erfahrung, die man auf keinen Fall noch einmal machen will. 1971 war die Studentenbewegung, die 1967 begonnen hatte, quantitativ wie qualitativ auf ihrem Höhepunkt. Am 12 März 1971 stellte das Militär dem Parlament ein 'Memorandum' zu, in dem es forderte, der damalige Ministerpräsident Demirel müsse zurücktreten - was der dann auch tat. Darauf bildete ein unabhängiges Parlamentsmitglied ein Kabinett von 'Technokraten', was ein völlig verfassungskonformer Vorgang war. Erst dann - und darin liegt der Unterschied zu 1980 - wurde das Kriegsrecht verkündet, um die sogenannten 'Anarchisten' (d.h. die Führer der Linken) zu verfolgen, die in ländlichen Regionen eine bewaffnete Guerillabewegung begonnen hatten. In den ersten beiden Monaten war damals die Repression recht milde. Am 12. März war die Linke in ihrer Gesamtheit (mit sehr wenigen Ausnahmen) für den Putsch, der als großer Rückschlag für die bürgerliche Regierung Demirel angesehen wurde. Einschließlich der größten Studentenorganisation Dev-Genc (Revolutionäre Jugend) waren alle für den Putsch. Natürlich mußten sie bald, als nämlich der Zugriff des Staates härter wurde, erheblich umlernen. Am 5. Mai tauchten die ersten Listen auf. Die meisten Führer gaben kampflos auf. Gewerkschaftsführer, Intellektuelle, Professoren usw. wurden verhaftet und vor Gericht gestellt. Man durchsuchte ihre Häuser und beschlagnahmte Bücher als 'Bewreise' für ihre Teilnabme an subversiven Aktivitäten. An diesem Punkt ging man aber entschieden zu weit. Diese Bücher waren nicht illegal oder gerichtlich verboten worden 4 Als Journalisten den Ministerpräsidenten danach fragten, sagte der im Fernsehen, daß er selber solche Bücher in seinem Haus hätte und daß es in der Türkei Ge- 
dankenfreiheit gebe. Die meisten Verhafteten wurden damals nach und nach wegen Mangels an Beweisen wieder entlassen. Die Washington Post (vom 15.9.80) formulierte es so: »Zwei mal zuvor - 1960 und 1971 - als sie einen Putsch starteten, brachten sie die zivilen Angelegenheiten völlig durcheinạnder und gaben die Macht bei der ersten passenden Gelegenheiten wieder an die Politiker zurück.«Dieses Mal werden die Militärs sicherlich vorsichtiger sein. Sie haben aus vergangenen Erfahrungen gelernt und sind entschlossen, die Struktur des Staates völlig umzugestalten, um zu verhindern, daß sich solche Situationen wiederholen.

Vor neun Jahren - das ist ein wichtiger Punkt - hat die Linke, sagen wir mal so, keine 'wirkliche' Repression zu spüren bekommen. Im äußersten Fall blieben die Leute zweieinhalb Jahre im Gefängnis, bis zur allgemeinen Amnestie von 1974. Jetzt sind die Bedingungen weitaus schlimmer geworden, und die Erfahrungen der letzten neun Monate zeigen, daß uns das Schlimmste noch bevorsteht. Andererseits ist die Lage ökonomisch gesehen nicht mit der von 1971 oder von 1980 zu vergleichen. 1971 war die wirtschaftliche Lage der Türkei recht günstig, es gab keine Devisenkrise, keine raketengleiche Inflation. 1980 hingegen liegen die Dinge ganz anders...

Mir ist immer noch nicht ganz klar, warum die Armee den Entschluß gefaßt bat, direkt in die Regierungsgeschäfte einzugreifen und das Risiko eines Sturzes der Demirel-Regierung auf sich zu nehmen - gerade wenn man diese früberen Erfabrungen berücksichtigt.

Auf die Gefahr hin, das Problem vereinfacht darzustellen, würde ich zwei Hauptgründe anführen: politische und ökonomische. Politisch waren die Militärs erschreckt über die terroristische Gewalt, die nicht einmal mithilfe des Kriegsrechts unter Kontrolle zu bringen war. In den letzten beiden Jahren hat es mehr als 5000 Fälle von politischem Mord gegeben. Es war eines ihrer erklärten Ziele, dem ein Ende zu bereiten. Aber sie waren durchaus auch nicht glücklich mit den Faschisten - der MHP - in Demirels Regierung. Obwohl man auch sagen kann, daß gewisse Kreise in der Armee damit sehr zufrieden waren. Aber der größte Grund ihrer Besorgnis war vermutlich die Tatsache, daß - abstrakt gesprochen - der Staat innerlich am Zusammenbrechen war. Das Parlament war durch Spaltungen und Streitigkeiten zerrissen. Evren sagte auf einer Pressekonferenz, die Politiker hätten »anstatt das Feuer gemeinsam zu löschen, Öl hineingegossen, weil es ihr einziges Ziel war, an die Macht zu kommen.«Die Regierung war schwach: Demirel mußte eine Minderheitsregierung mit der MHP und der MSP bilden und wurde vom Parlament ständig daran gehindert, das Programm dieser Regierung durchzuführen; wohingegen Evren und die höheren Offiziere als Lösung für die Probleme des Landes von einer 'Nationalen Union' zwischen den beiden großen Parteien träumten, Demirels Gerechtigkeitspartei und Ecevits Republikanischer Volkspartei. In seinen Reden nach dem Staatsstreich äußerte Evren scharfe Kritik an den Politikern, denen er vorwarf, nur ihre Wahlziele verfolgt zu haben. Zu beachten ist dabei, daß die Armee sich als Hüterin der republikanischen, verfassungsmäßigen und laizistischen Prinzipien von Atatürk betrachtet, welche die Vorstellung von einem starken Staat einschließen und wenig Raum für die Demokratie lassen. Dieser subjektive Faktor ist, denke ich, sehr wichtig, um zu verstehen, wie sie ihr Handeln legitimieren.

Insbesondere waren sie sehr entsetzt über die Taktiken von Erbakans Nationaler Heilspattei, einer recht kleinen politischen Gruppierung mit stark islamisch-fundamentalistischer Ideologie. Erbakan stand der Koalitionsregierung, wiewohl seine Partei an ihr beteiligt war, äußerst kritisch gegenüber. So stimmten seine Abgeordneten beim letzten Vertrauensvotum, das mit dem Rücktritt des Außenministers endete, gegen die Regierung. Ande- 
rerseits weigerten sich die Aktivisten seiner Partei, die Nationalhymne zu singen, was für die Militärs eine große Beleidigung darstellt. Die Nationale Heilspartei, die - fast im Stile der Weimarer Republik - einen politischen Einfluß hat, der ihrer Stärke nicht entspricht, ist in der Tat eine sehr merkwürdige Gruppierung. Obwohl sie eine rechte islamische Ideologie vertritt, waren ihre Abgeordneten die einzigen, die vor einigen Monaten im Parlament gegen die Repressions-Gesetze gestimmt haben, wogegen die Republikanische Partei, die eigentlich sozialdemokratisch ist, dafür gestimmt hat.

"Meinst du, die Armee hat die Macht übernommen, weil sie womöglich einen allgemeinen Aufstand befürchtet hat?

Ganz sicher nicht. In der aktuellen Situation gab es keine Alternative, weder auf der Rechten noch auf der Linken. Die Linke war in mehr als 60 verschiedene Gruppen aufgesplittert und war darauf in keiner Weise vorbereitet. Die Rechte war auch nicht gut organisiert, wenn sie auch sehr an Stärke zugenommen hatte. Ihr Ziel war es aber nicht, an die Macht zu kommen, sondern Städte und Gebiete unter ihre Kontrolle zu bringen (zu 'befreien'). Angesichts dieser Lage ist das Militär sicher darauf aus, mit allen physischen und anderen Mitteln die Wurzeln dieser Ideologien zu vernichten, betrachtet sie diese doch - zusammen mit der Unfähigkeit der Politiker zum Regieren - als Mutter aller Übel.

Wie steht es mit der ökonomischen Situation? Du bast vorbin gesagt, die sei der andere Grund für den Putsch gewesen. In welchem Sinne?

Die Türkei steckte in der - wie Demirel sich ausdrückte - sschlimmsten Krise in der Geschichte des Landes«, was der Wahrheit sehr nahekommt. Nach einer Periode relativ erfolgreicher Entwicklung - begleitet von einer massiven Auswanderung türkischer Arbeiter in die westeuropäischen Länder - in welcher der Staat eine entscheidende Rolle spielte, wurde die Türkei in den letzten sieben Jahren von der internationalen Wirtschaftskrise hart getroffen und versucht nun, sich im Rahmen der neuen internationalen Arbeitsteilung auf dem Weltmarkt zurechtzufinden. Zunächst stellte der Preisanstieg für Ölimporte, von denen die Türkei hundertprozentig abhängig ist, das Land vor sehr ennste Zahlungsbilanzprobleme. Die Intervention in Zypern und die darauffolgenden Embargomaßnahmen der USA haben für die ökonomische Lage ebenfalls schwere Folgen gehabt. Die Türkei ist ein bedeutender Exporteur von Textilien, hat aber neuerdings infolge der britischen Konkurrenz Schwierigkeiten, diese in der EG abzusetzen. Im zweiten Halbjahr 1980 bewegte sich das Defizit der Handelsbilanz in einer Größenordnung von 1,3 Milliarden Dollar, und die Importe nahmen trotz Importbeschränkungen viel schneller zu als die Exporte. Die 'Gastarbeiter' hörten auf, ihr Geld in die Türkei zurückzuschicken und legten es lieber auf westdeutschen Bankkonten fest.

Die Türkei hat mit die höchsten Auslandsschulden in der Welt, sie werden offiziell auf 14,6 Milliarden Dollar geschätzt, belaufen sich wahrscheinlich aber auf 18 Milliarden. Kapitalistische Länder, besonders Westdeutschland, das in jüngster Zeit die USA in der Rolle des »reichen Onkels« abgelöst hat, haben für die Türkei im Hinblick auf deren strategische, ökonomische und militärische Lage ein 'Hilfsprogramm' aufgestellt: zwei Milliarden Dollar für die Jahre 1979 und 1980. Deutschland finanziert in der Türkei auch umfangreiche Projekte von Atomkraftwerken.

Wie immer bei Ländern der Dritten Welt, deren ökonomische Entwicklung von den imperialistischen Ländern überwacht werden muß, ist diese 'Hilfe' an die Übernahme eines Austerity-Programms gebunden, das der Türkei vom Weltwährungsfond (IMF) auferlegt wird, der als internationaler Finanzgendarm agiert. Der IMF hat im Januar 1980 mit der 
Demirel-Regierung die Umschuldung der Auslandskredite ausgehandelt und dem Land ein hartes Programm verpaßt, das darauf zielte, die dreistellige Inflationsrate zu reduzieren, das Staatsdefizit zu kontrollieren und die Zahlungsbilanz zu stabilisieren. Dazu wurden Preiskontrollen aufgehoben - vor allem auch die der Zinsraten, die jetzt auf $50 \%$ angestiegen sind - Subsidien gekürzt (hauptsächlich für Düngemittel, die für die türkische Landwirtschaft ein wesentlicher Faktor sind) und Maßnahmen zur Kontrolle der Löhne eingeführt.

Es mag überraschen, daß die Militärregierung als eine ihrer ersten Handlungen das IMFProgramm, das die Demirel-Regierung zu verwirklichen begonnen hatte, in vollem Umfang gutgeheißen und angekündigt hat, die Türkei werde all ihre finanziellen Verpflichtungen erfüllen. Dies liegt aber in Wahrheit völlig in der Logik des Kapitalismus. Mit einer Minderheitsregierung war es praktisch unmöglich, ein solches Programm durchzuführen. Im März 1980 war die Haushaltsdebatte im Parlament bereits ein erster Test für dieses Programm gewesen: das Budget wurde mit einer nur sehr knappen Mehrheit verabschiedet. Wichtiger noch: es war klar, daß die Durchführung des Austerity-Programms durch die Arbeiterklasse blockiert worden wäre. Für September waren wichtige Streikaktionen angekündigt, die von der Regierung mithilfe ihrer verfassungsmäßigen Vollmachten zeitweise unterbunden wurden. In der Türkei sind zwei Millionen Arbeiter gewerkschaftlich organisiert und die Regierung kann seit dem Gesetz von 1964 zwar Streiks in lebenswichtigen Industriezweigen drei Monate lang aufschieben, nicht aber legalerweise in Tarifauseinandersetzungen eingreifen.

Es ist klar, daß eine starke Militärregierung, die exekutive und legislative Macht in einer Hand konzentriert, diese Hindernisse beseitigen und das IMF-Programm durchziehen kann. Der amtierende Ministerpräsident, der wenige Tage nach dem Putsch vom 'Nationalen Sicherheitsrat' ernannt wurde - Turgut Ozal, ein ehemaliger Mitarbeiter des IMF in Washington und seit ein paar Jahren der türkische 'Finanz-Zauberer', der das volle Vertrauen der internationalen Finanzkreise genießt - ist in der neuen, von der Junta eingesetzten Ulusu-Regierung zum stellvertretenden Ministerpräsidenten mit Zuständigkeit für die Wirtschaft ernannt worden. Mit der Auflösung der zwei großen Gewerkschaftsverbände, dem Verbot von Streiks und der Möglichkeit, 'uneffektive' Staatsbeamte zu feuern, ist klar geworden, daß das IMF-Programm jetzt reibungsloser durchgeführt wird. Wenige Tage nach dem 12. September, während Evren noch die 'agas' der Gewerkschaften denunzierte, die angeblich die Arbeiter »mißbraucht und manipuliert《 hätten, wurden schon neue Preiserhöhungen (u.a. für Öl, Zucker und Düngemittel) angekündigt.

Noch eine Frage: Wer ist für den Putsch verantwortlich? Kann man sagen, daß er ein Resultat der Schwäche der Linken ist?

So hart würde ich das nicht sagen. Aber offensichtlich ist der Putsch, wenn auch nicht eine direkte Folge, so doch teilweise ein Ergebnis der Fehler der Republikanischen Partei - und auch der Unfähigkeit der Linken, eine stimmige Alternative zu Ecevits sozialdemokratischer Politik anzubieten.

Ecevit stellte für Demirel keine ernsthafte Opposition dar. In vielen Punkten hatten sie dasselbe Programm. Als Ministerpräsident hatte Ecevit handfester gesprochen als er dańn in Wirklichkeit handelte. Das führte lediglich dazu, daß ihm die Geschäftsleute mißtrauten und die Arbeiter ihre Hoffnungen begruben. Er war mehr mit den internen Konflikten seiner eigenen Partei beschäftigt als mit den Geschehnissen außerhalb. Man könnte fast sagen, daß Demirels Auftreten 'demokratischer' erschien als Ecevits. Demirel legte großen 
Wert auf sein offentliches Image und erschien jeden Abend auf dem Bildschirm, um zu erläutern, welche Maßnahmen er ergreifen wollte.

Ecevit hat in den letzten Jahren so gehandelt, als könne er ganz sicher auf die Unterstützung der Linken zählen, die ihm zunehmend mißtraute und von ihm abfiel. Ecevit verlegte sich darauf, Wählerstimmen auf der Rechten zu gewinnen, um diesen Einfluß auszubalancieren. Die türkische Linke ist sicher nicht mit der italienischen zu vergleichen, aber sie konnte die Republikanische Partei an die Macht bringen und zum Rücktritt zwingen, indem sie ihr die Unterstützung entzog. Aber obwohl sie diesen Einfluß seit den Wahlen von 1973 hatte, war sie nicht in der Lage, eine populäre Alternative zu Ecevit aufzubauen. Die Linke in der Türkei ist extrem zersplittert, was wohl die verschiedenartigen und komplexen Unterschiede widerspiegelt, die in den fortschrittlichen Teilen der Bevölkerung hinsichtlich Klassenlage, regionaler Herkunft und geistiger Orientierung anzutreffen sind. Ein verbreiteter Witz lautete, daß es unmöglich sei, alle linken Organisationen aufzuzählen, denn kaum habe man sie durch, sei schon wieder eine neue entstanden.

Einer der Grundzüge der politischen Szene war - jenseits der Militanz in den Gewerkschaften, Lehrerorganisationen usw. - die Existenz von 'befreiten Gebieten' in den wichtigsten Städten des Landes, d.h. von Basisorganisationen. Linke Gruppen begannen (etwa seit Mitte der 70er-Jahre), die Bevölkerung gegen faschistische Úbergriffe, die eine echte Bedrohung darstellten, zu schützen und Nahrungsmittel-Kooperative zu organisieren, die der ökonomischen Krise begegnen halfen. In den Gecekondus (Slumartige Siedlungen von improvisierten Häusern in den Randzonen der Großstädte, d.Ü.) halfen sie den Leuten, sich niederzulassen, Baumaterial zu beschaffen, Siedlungspläne zu entwickeln und die Bewohner zu schützen - sie nahmen also Aufgaben wahr, die der Staat wahrzunehmen sich weigerte. In meinen Augen war dies die wichtigste Errungenschaft der Linken: eine embryonale Organisation des Volkes, wenn diese auch hauptsächlich defensiv war und nicht in einen Einigungsprozeß mündete.

In den letzten Jahren begannen sich auch 'befreite Zonen' der Faschisten in den Städten auszubreiten, vor allem in Ost- und Zentralanatolien, aber auch in Istanbul, Ankara usw. Bestimmte Städte waren völlig von Faschisten beherrscht, was die progressiven Teile der Bevölkerung dazu zwang, sich in bestimmten Gebieten niederzulassen, um sich eine Verteidigungsorganisation zu schaffen. Das führte zu einer derartigen Polarisierung, daß viele Leute vom rechten Flügel der CHP den Schutz linker Organisationen in Anspruch nehmen mußten, nur um zu überleben. Die CHP selber unternahm in dieser Hinsicht gar nichts. Was wird nach dem Putsch aus der Linken? Wird sie den bewaffneten Kampf fortsetzen? Ich glaube nicht. Die Gruppen, die das versucht haben, sind schonungslos verfolgt worden. Ich fürchte, daß von ihnen nichts übrigbleiben wird. Aber der andere Teil der Linken ist zahlenmäßig zu stark, um ohne weiteres unterdrückt zu werden. Man kann nicht eine ganze Bevölkerung einfach auslöschen. In den nächsten Monaten wird die Linke vermutlich eine abwartende Haltung einnehmen und überdies alle Hände voll zu tun haben, um ihre bloße Existenz zu erhalten. Man kann jetzt noch nicht sagen, in welche Richtung die neuen Enswicklungen treiben werden. Eins aber ist sicher: die Militärs werden ganz neue politische Bedingungen setzen, die Vetfassung, die Repressionsgesetze ändern, die breiten Volksmassen vollends ökonomisch in die Zange nehmen. Das wird für die Linke in der Tür* kei der Beginn einer neuen Periode sein. 